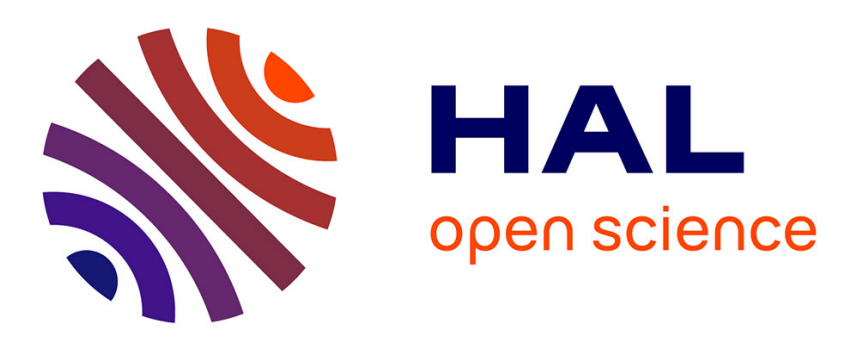

\title{
Study of conduction and induction phenomena in electric circuits using a time-domain integral formulation
}

François Bost, Laurent Nicolas, Gérard Rojat

\section{To cite this version:}

François Bost, Laurent Nicolas, Gérard Rojat. Study of conduction and induction phenomena in electric circuits using a time-domain integral formulation. IEEE Transactions on Magnetics, 2000, 36 (4 Part 1), pp.960-963. hal-00140554

\section{HAL Id: hal-00140554 https://hal.science/hal-00140554}

Submitted on 18 Apr 2007

HAL is a multi-disciplinary open access archive for the deposit and dissemination of scientific research documents, whether they are published or not. The documents may come from teaching and research institutions in France or abroad, or from public or private research centers.
L'archive ouverte pluridisciplinaire HAL, est destinée au dépôt et à la diffusion de documents scientifiques de niveau recherche, publiés ou non, émanant des établissements d'enseignement et de recherche français ou étrangers, des laboratoires publics ou privés. 


\title{
Study of Conduction and Induction Phenomena in Electric Circuits Using a Time-Domain Integral Formulation
}

\author{
François Bost, Laurent Nicolas, and Gérard Rojat
}

\begin{abstract}
This paper deals with the modeling of the susceptibility of electric circuits. A thin-wire time-domain integral formulation based on the antenna theory is used to calculate both conducted current due to a voltage generator and induced current due to an electromagnetic perturbation. Special numerical treatment is used for angular points and multiple junctions in the electric circuit. Voltage source can be inserted in the model. Non linear components, such as diodes or transistors, are described under their high frequency behavior.
\end{abstract}

Index Terms-Wire scatterers, time domain analysis, integral equations, numerical analysis.

\section{INTRODUCTION}

$\mathbf{T}$ HE calculation of the electromagnetic susceptibility of an electric circuit without taking into account its nominal operating conditions is not really significant. On the other hand the superposition of both electromagnetic phenomena-conducted and radiated - is important in order to determine the behavior of the electric circuit when illuminated by an electromagnetic perturbation. The main difficulty lies then in superimposing both conducted current due to a voltage generator and induced current due to the perturbation. To our knowledge, no such attempt has been made previously.

A review of time-domain methods used in electromagnetics has been given in [1]. Since we are mainly concerned by thin wire devices, an integral equation formulation seems very attractive. Several implementations of a such a method have been published, especially for volume problems and for the magnetic field integral equation [2]. However, because of the presence of thin wires, only the electric field integral equation (EFIE) may be useful [3]. In [4], a time-domain model developed in order to provides the transient response of a complex 3D wire structure is presented. Our purpose is to use the same formulation in order to modelize both induced and conducted problems, so that the analysis of the susceptibility of an electronic device in normal running is made possible.

For such a purpose, three major difficulties have to be solved. First, the conductive wires can be connected each others with

Manuscript received October 25, 1999. This work was supported in part by the région Rhöne-Alpes (France).

F. Bost and L. Nicolas are with the CEGELY, UPRESA CNRS 5005, Ecole Centrale de Lyon, BP 163, 69131 Ecully cedex, France (e-mail: bost@eea.eclyon.fr, laurent@eea.ec-lyon.fr).

G. Rojat is with the CEGELY, UPRESA CNRS 5005, Universite Claude Bernard, Bât. 721, 43 bld du 11 novembre 1918, 69622 Villeurbanne, France (e-mail: rojat@cegely.univ-lyon1.fr).

Publisher Item Identifier S 0018-9464(00)05645-4. multiple junctions. Second, generators may be inserted in the conductive structure. Third, the conductors may be loaded with non linear electronic components. All these points are discussed in the following sections. In conclusion, two examples of simulation show the capabilities of the formulation.

\section{The Thin-Wire Time-DOMAIN INTEGRAL FormULATION}

The thin-wire time-domain integral formulation is deduced from the classical EFIE. It is obtained with the assumptions that the scatterers are perfect conductors and that the current density may be restricted to its tangential term. It is then written as [4]:

$$
\begin{aligned}
\mathbf{t}^{\prime} \cdot \mathbf{E}^{i n c}\left(x^{\prime}, t\right) & \\
= & \frac{\mu_{0}}{4 \pi} \int_{L}\left[\frac{\mathbf{t}^{\prime} \cdot \mathbf{t}}{R} \frac{\partial}{\partial \tau} I(x, \tau)+c \frac{\mathbf{t}^{\prime} \cdot \mathbf{R}}{R^{2}} \frac{\partial}{\partial x} I(x, \tau)\right. \\
& \left.+c^{2} \frac{\mathbf{t}^{\prime} \cdot \mathbf{R}}{R^{3}} \int_{-\infty}^{\tau} \frac{\partial}{\partial x} I(x, t) d t\right] d x
\end{aligned}
$$

where $\mathbf{E}^{\text {inc }}$ is the incident field, $I$ is the current in the wire, $\mathbf{t}$ is the tangent vector to the wire at the computation point, $\mathbf{t}^{\prime}$ is the tangent vector at the collocation point, $R$ is the distance between both of these points, $\tau=t-R / c$ is the retarded time (Fig. 1).

\section{A. Numerical Solving of the Thin-Wire Time-Domain EFIE}

This integral formulation is solved by the Method of Moments. The current is described by a first order expanding scheme. The delta function is used as testing function for point-matching. The unknown current at the observation time is then obtained by solving an implicit time scheme [4]. The final matrix system may be written symbolically as:

$$
\mathbf{E}_{M k}^{\text {inc }}=Z_{M I} \cdot \mathbf{I}_{I k}+\mathbf{E}_{M j}^{\text {sca }} \quad \text { for } j=1, k-1
$$

where $\mathbf{E}_{M k}^{\text {inc }}$ is the incident field vector tangent at point $M$ and time $t_{k}, Z_{M I}$ is the geometrical matrix of the elementary contributions between nodes $M$ and $I, \mathbf{I}_{I k}$ is the current vector at node $I$ and time $t_{k}$, and $\mathbf{E}_{M j}^{\text {sca }}$ is the scattered field vector tangent at point $M$ depending on the earlier currents at times $t_{j}$ (Fig. 1).

The stability and the accuracy of the scheme depend on the characteristic parameters of the wire and on the space and time discretization. It has been shown that the optimal values for a $L$ length wire and a $\lambda$ wavelength illumination are:

- Radius of the wire: $a \ll L$ and $a \ll \lambda$,

- Space discretization: $\Delta l=5 a$, with $\Delta l \leq \lambda / 10$,

- Time discretization: $\Delta t=\Delta l / c$ to $4 \Delta l / c$, with $\Delta t \leq$ $\lambda / 10 c$. 


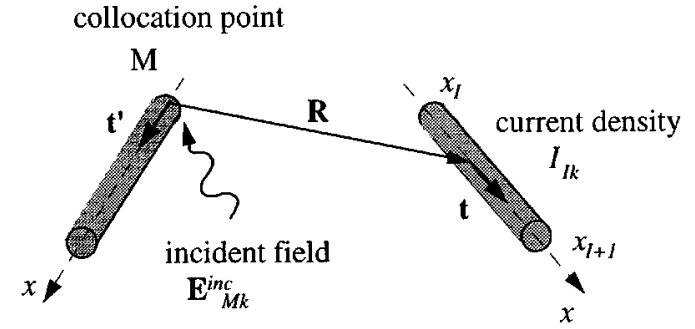

Fig. 1. Contribution of a straight segment at the collocation point $M$.

\section{B. Ability for the Model to Handle Conducted Currents}

By using the EFIE, the scattered electric field due to the induced currents is computed at any point. If the electric field is calculated from the global contribution of the currents in all the circuit, the modeling may be seen as general, since the radiation of all the circuit is taken into account: it is a classical EMC problem. On the other hand, if the electric field is calculated only from the localized contribution of the currents in the adjacent segments, the modeling becomes circuit-like. In other words, by coupling only the adjacent segments, the energy is transmitted by going through the wires, and the formulation can then handle pure conducted phenomena.

\section{Charge Distribution And Multiple Junctions}

Singular geometric points are defined as points where the geometry varies abruptly. They are of three kinds: free extremities, angular points and multiple junctions. Free extremities are easy to handle with, since it is sufficient to apply the appropriate boundary condition. On the other hand, special numerical treatment is used for angular points and multiple junctions.

\section{A. Case of the Angular Points}

The choice of the basis functions has a great influence on the representation of the physical phenomenon. Since a first order scheme is used for the current, the charge distribution is assumed to be constant along each discretization segment. So the continuity is not applied to the charge density along the wire, and a charge accumulation can be observed in some case on a straight segment. The distribution of charge depends actually on the relative values of the coupling terms between the adjacent segments, i.e. the charges are divided up in the same proportion as the coupling values. The coupling terms are directly related to the inner product $\mathbf{t}^{\prime} \cdot \mathbf{t}$, where $\mathbf{t}^{\prime}$ and $\mathbf{t}$ are respectively the tangent vectors at the collocation point and at the current point of the contributive segment (Fig. 1). It becomes then sufficient to make the structure symmetrical at angular points by calculating an average tangent at the collocation point (Fig. 2).

The electric circuit shown in the Fig. 3 is used to validate this numerical treatment. The circuit is discretized with 16 segments, the space discretization is $\Delta l=3 \mathrm{~cm}$. Values of the resistance and the voltage source are respectively $R=100 \Omega$ and $E=100 \mathrm{~V}$. Two configurations are studied: with correction of the tangent at angular collocation points (nodes \#7 and \#11), and without correction. As shown in Fig. 4, the distribution of the charges is accurate only if the tangent at the angular points

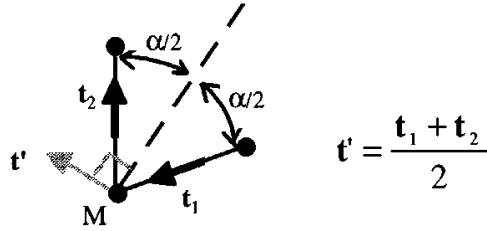

Fig. 2. Tangent at the collocation point $M$ for an angular point.
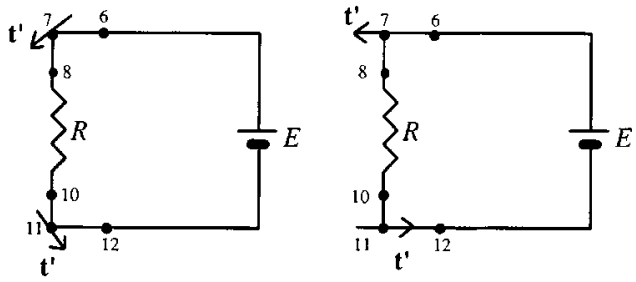

Fig. 3. Comparison of the 2 configurations used to validate the correction of the tangent at angular points. Left: with correction. Right: without correction.

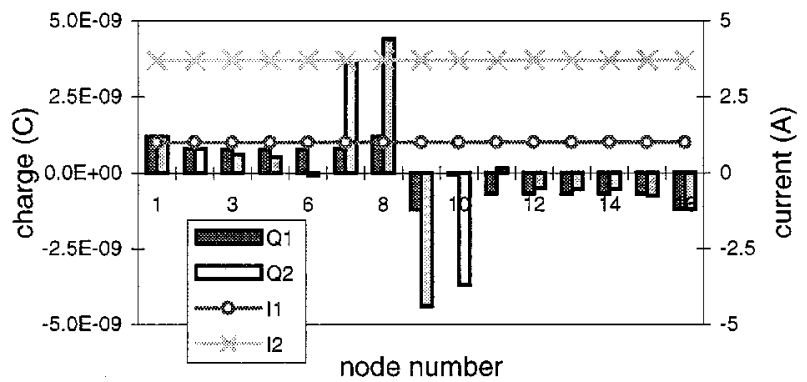

Fig. 4. Distribution of the charge $Q$ and the current $I$ along the electric circuits for both configurations. $Q 1$ and $I 1$ are computed with tangent correction, $Q 2$ and $I 2$ are computed without correction.

is corrected, since a discontinuity appears in this case only at node \#1 and node \#9 corresponding to the generator and the resistance. The magnitude of the current is then in good agreement (equal to $1 \mathrm{~A}$ ).

\section{B. Case of Multiple Junctions}

A multiple junction is also seen as a singularity. In this case, it is not possible to define an unique tangent vector $\mathbf{t}^{\prime}$ at the junction node. The first order scheme used to describe the current affects also the Kirchhoff's laws on the currents and voltages. The junction is then first made symmetric, in order to obtain identical values for the inner product $\mathbf{t}^{\prime} \cdot \mathbf{t}$. Secondly, it is replaced by as many nodes as connected segments (Fig. 5), and the Kirchhoff's law written at the junction is inserted in the matrix system.

Fig. 5 shows the case where 3 segments are connected: the angle between the segments is equal to $120^{\circ}$, and the value of the inner product $\mathbf{t}^{\prime} \cdot \mathbf{t}$ is equal to $\cos \left(120^{\circ}\right)=1 / 2$. The case of the junction between $N$ segments is ten deduced by recursive process (Table I).

This model of junction may be validated analytically. The example shown at Fig. 6 presents a configuration of electric circuit with 3 junctions (nodes \#3, \#5 and \#13). Computed values of the current are in good agreement with analytical values. 

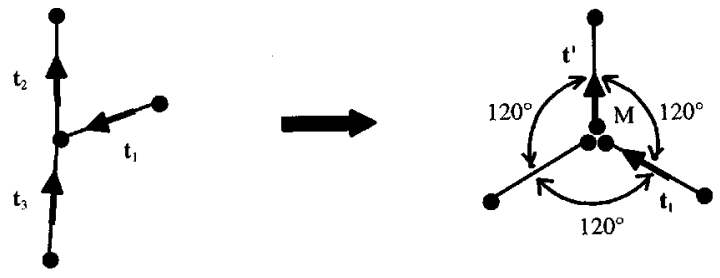

Fig. 5. Tangent at the collocation point $M$ for a 3 segments junction.

TABLE I

SYMMETRIZATION OF THE JUNCTION: VALUES OF THE INNER PRODUCTS DEPENDING ON THE NUMBER OF CONNECTED SEGMENTS

\begin{tabular}{ccc}
\hline $\begin{array}{c}\text { Number of } \\
\text { connected segments }\end{array}$ & $\begin{array}{c}\text { Value of the inner } \\
\text { product } t^{\prime}, t_{i}\end{array}$ & $\begin{array}{c}\text { Angle between the segment } \\
\text { (after symmetrization) }\end{array}$ \\
\hline 2 & 1 & $180^{\circ}$ \\
3 & $1 / 2$ & $120^{\circ}$ \\
4 & $1 / 3$ & $109.47^{\circ}$ \\
$\mathrm{N}$ & $1 /(\mathrm{N}-1)$ & $\mathrm{A} \cos [1 /(\mathrm{N}-1)]$ \\
\hline
\end{tabular}

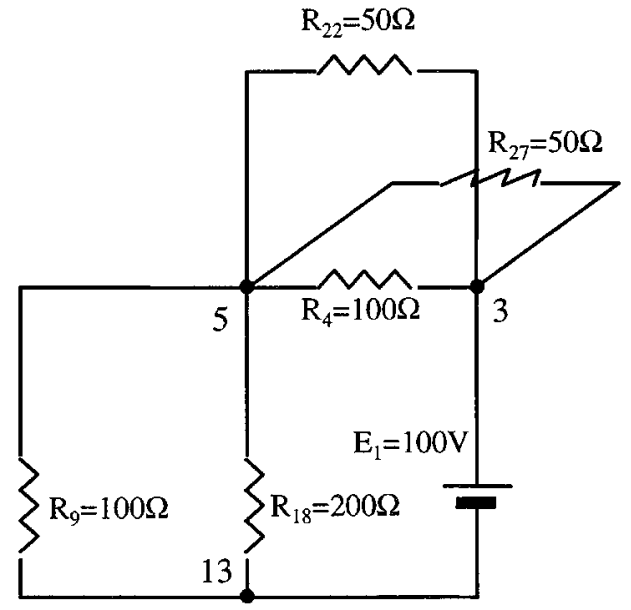

Fig. 6. Validation of the model of multiple junctions. Analytical and computed values of the current in the voltage source $\left(I_{1}\right)$ and in the resistances.

\section{INSERTION OF VOLTAGE SOURCES}

A voltage generator is defined as a local source of radiation, i.e. as an additional electric field $\mathbf{E}^{\prime}$, for which an integration on a closed loop is not zero. With first order basis functions, the voltage is related to the electric field at a node $M$ by using:

$$
\begin{aligned}
U_{M} & =\int_{x_{M-1}+x_{M} / 2}^{x_{M+1}+x_{M} / 2} E(x, t) d x \\
& \approx \frac{1}{2}\left(x_{M+1}-x_{M-1}\right) \cdot E_{M}^{\prime}(t)
\end{aligned}
$$

\section{INSERTION OF NON LINEAR COMPONENTS}

Any component can be taken into account in the model, whether linear or not, if its insertion respects several rules. First, components are inserted at the nodes of the structure. Second, they are described in their transient form. Third, their model simulate the real high frequency behavior.

\section{A. Nodal Insertion}

By assuming that the dimension of a component is not significant, its self susceptibility and self radiation are not modeled: a component is reduced to a nodal contribution.

\section{B. Time-Domain Formulation}

A component is characterized by its transient impedance. If non linear, the impedance has to be calculated at each time step. The relation between the electric field $E_{i}$ at node $i$ and the "nodal" voltage Ui across a segment centered at the node is written using (2). The Ohm's law is used to describe the behavior of the component. In the general case, with the previous notations, it may be written in matrix form as:

$$
\mathbf{U}_{M k}=\mathbf{Z}_{M}^{k} \mathbf{I}_{l k}+\sum_{j=0}^{k-1} \mathbf{Z}_{M}^{j} \mathbf{I}_{i j}
$$

where $\mathbf{Z}_{j M}$ is the impedance of the component located at node $M$ and at time $t_{j}$. For the $R, L$ and $C$ components, it can be written as:

$$
\begin{aligned}
\text { Resistance: } \quad \mathbf{U}_{M k} & =R_{M} \mathbf{I}_{M k} \\
\text { Inductance: } \quad \mathbf{U}_{M k} & =\frac{L_{M}}{\Delta t} \mathbf{I}_{M k}-\frac{L_{M}}{\Delta t} \mathbf{I}_{M k-1} \\
\text { Capacity: } \quad \mathbf{U}_{M k}= & \frac{\Delta t}{C_{M}} \mathbf{I}_{M k}+\frac{\Delta t}{C_{M}} \mathbf{I}_{M k-1} \\
& +\frac{\Delta t}{C_{M}} \sum_{j=1}^{k-1}\left(\mathbf{I}_{M j}+\mathbf{I}_{M j-1}\right)
\end{aligned}
$$
(3):

The final electric field equation is obtained by adding (1) and

$$
\begin{aligned}
\mathbf{E}_{M k}^{\mathrm{inc}}+\frac{\mathbf{U}_{M k}}{\Delta l_{M}}= & \left(\mathbf{Z}_{M I}+\frac{\mathbf{Z}_{M}^{k}}{\Delta l_{M}}\right) \mathbf{I}_{l k}+\mathbf{E}_{M j}^{\mathrm{sca}} \\
& +\frac{1}{\Delta l_{M}} \sum_{j=0}^{k-1} \mathbf{Z}_{M}^{j} \mathbf{I}_{i j}
\end{aligned}
$$

where $\Delta l_{M}$ is the half-length of the segment $\left[x_{M-1}, x_{M}\right]$. Note that the matrix $\mathbf{Z}_{M I}$ remains constant during the solving, since it is only due to the geometry of the electric circuit. On the other hand, the matrix $\mathbf{Z}_{M}^{k}$ has to be generally updated at each time step.

\section{High Frequency Behavior}

The frequencies and the magnitudes of the radiated fields depend actually strongly on the parasitic elements of a component. For example a parasitic capacity in parallel with a high inductance may develop high frequency resonance, which are of great importance for an EMC purpose. So a component has to be described with a detailed model such as those found in [5] or [6]. Fig. 7 shows as example the model of the diode used in the following section.

\section{EXAMPLES OF SimUlation}

\section{A. Illumination of a Mono-Alternance Rectifier}

Figs. 8 and 9 show the current in a single loop loaded with a soft diode [5] and a $100 \Omega$ resistance, in normal running 


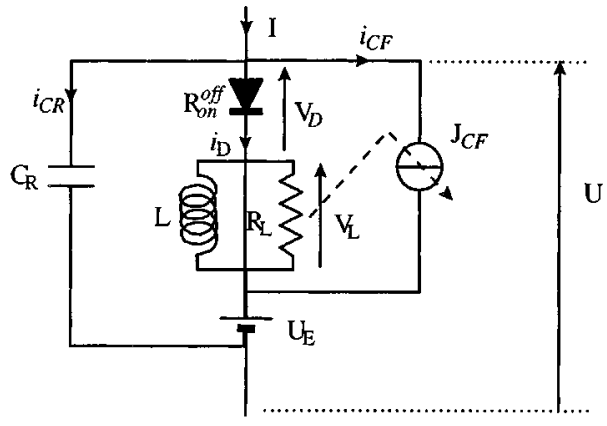

Fig. 7. Detailed model of the diode [1].

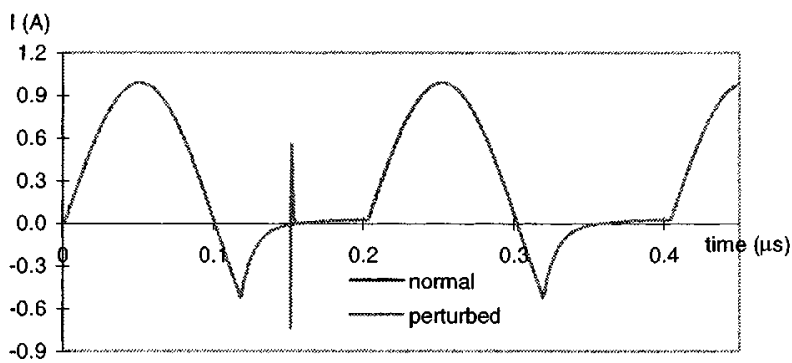

Fig. 8. Current in a single loop rectifier: normal and perturbed currents are exactly superimposed, except during the pulse at time $0.15 \mu \mathrm{s}$.

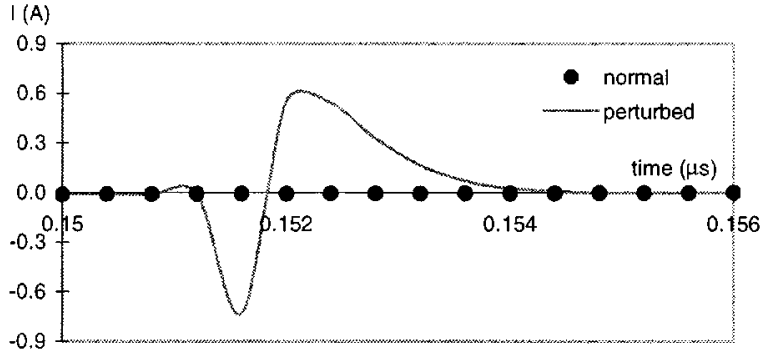

Fig. 9. Zoom of the previous curve around $0.15 \mu \mathrm{s}$.

and when illuminated by an electromagnetic perturbation. The source supplies a $100 \mathrm{~V}$ alternating voltage. In normal running, one can observe a large reverse recovering current due to the fact that it is a power diode. The biexponential plane wave perturbs the circuit at $0.15 \mu \mathrm{s}$. Since the loop is small and the decay time of the pulse is large, normal and perturbed current are superimposed except during the pulse: only its leading edge is observed.

\section{B. Illumination of a Distribution Network}

As shown in Fig. 10 the distribution network is modeled in a very simplistic way, in order to demonstrate the potentiality of the formulation. The network is made of a $\mathrm{AC}$ voltage generator with its internal resistance $r$ and by a resistance $R$ protected by a surge arrester. It is assumed that only the generator branch is illuminated by a lightning stroke defined by a biexponential. The dynamic model of the surge arrester is found in [6]. The conducting ground plane is modeled by applying the image theory.

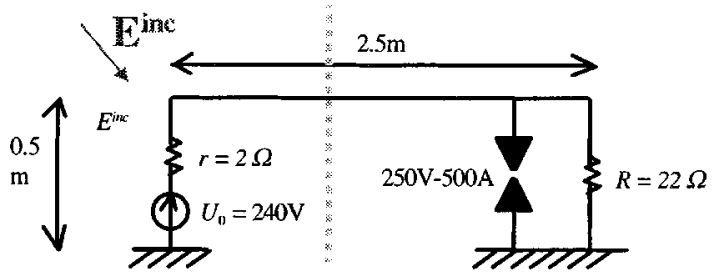

Fig. 10. Modeling of the distribution network.

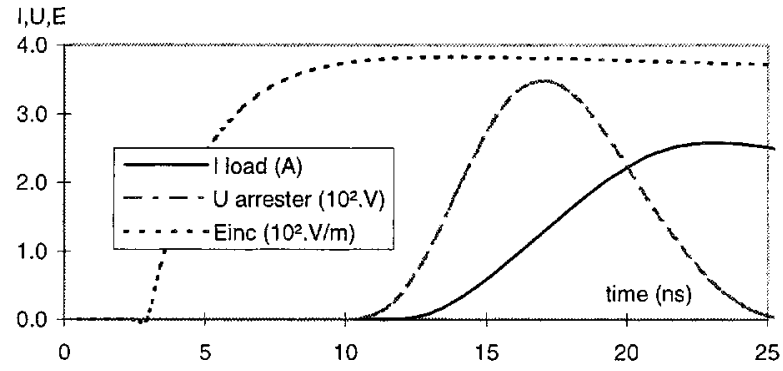

Fig. 11. Illumination of the network: pulse in the generator branch, arrester voltage and current in the load.

Fig. 11 shows the efficiency of the arrester, since the current in the load induced by the pulse is limited as soon as the surge arrester becomes conducting. Note that, due to the time constant of the pulse, the regular sinusoidal variation due the generator can not be observed (its periodicity is $50 \mathrm{~ms}$, compared to time constant of the pulse defined in ns!).

\section{CONCLusion AND Perspectives}

In this paper, a new transient model to evaluate at the same time both induced and conducted currents in wire circuits is presented. It is based on a coupling formulation between radiated fields and wires. It is shown how to deal with multiple junctions and to insert electric components and generators. This new formulation allows to study the susceptibility of electric circuits loaded with non linear components in operation.

Further developments have now to be made. First, it is necessary to validate the formulation with an adapted experimentation, since no numerical comparisons are available. Second, the formulation has to be optimized from a numerical point of view. Third, the thin-wire model has to be coupled with a boundary element model in order to modelize the environment.

\section{REFERENCES}

[1] E. K. Miller, "A selective survey of computational electromagnetics," IEEE Trans. Antennas Prop., vol. 36, pp. 1281-1305, Sept. 1988.

[2] M. J. Black and S. P. Walker, "Time-domain BIE analysis of large three-dimensional electromagnetic scattering problems," IEEE Trans. Antennas Prop., vol. 45, no. 5, pp. 894-901, May 1997.

[3] L. B. Felsen, Transient Electromagnetic Fields: Springer-Verlag, 1976.

[4] F. Bost, G. Rojat, and L. Nicolas, A time-domain integral formulation for the scattering by thin wires, in COMPUMAG'99, Sapporo, Japan, pp. $268-269,1999$.

[5] C. Batard, "Interactions composants-circuits dans les onduleurs de tension. Caractérization-Modélization-Simulation," Ph.D., INPT, Toulouse, 1992.

[6] P. Riaublanc and M. Renard, Modélization physique des éclateurs à gaz, in CEM'92, Lyon, France, pp. 420-426, 1992. 